\title{
Active filter synthesis based on nodal admittance matrix expansion
}

\author{
Lingling Tan, Yunpeng Wang and Guizhen $\mathrm{Yu}^{*}$
}

\begin{abstract}
Active network synthesis is important for circuit designer to find new circuits with desired performance. In this paper, a method of Tow-Thomas (TT) Bi-quad band-pass filter circuit generation methods is proposed using nullor representation of the operational amplifier (OPA), and a method for synthesizing active band-stop filters is presented, both of which start from voltage transfer function and linked infinity variables to describe nullors in both nodal admittance matrix (NAM) and port admittance matrix of the circuit to be synthesized. The Tow-Thomas band-stop filter circuit and Åkerberg-Mossberg band-stop filter circuit are synthesized by nodal admittance matrix expansion on the same port admittance matrix.
\end{abstract}

Keywords: Active filter synthesis, Nullor, Nodal admittance matrix (NAM) expansion, Band-pass filter, Band-stop filter

\section{Introduction}

Active network synthesis is the reverse process of the traditional active network analysis. Method of circuit synthesis makes circuit automatic design realizable [1]. Admittance matrices of the active devices, such as the ideal operational amplifier (OPA), current mirror, voltage mirror [2], do not exist. Through decades of painstaking research, D.G. Haigh and A.M. Soliman enrich the theory of active network synthesis. D.G. Haigh proposed the method of nodal admittance matrix (NAM) expansion and put it into practical applications, for example, obtaining the topology of a circuit from a given transfer function based on the theory of nullors [3]. Alternative circuit topology structures can be obtained from the same transfer function, when taking account of the different performances [4]. On the basis of the nullors, two additional pathological elements, current mirror (CM) and voltage mirror (VM) [5], are proposed, which tremendously enrich the theory of active network synthesis and make the active circuit design through theoretical method possible [6].

In comparison with the conventional generation methods of circuits based on experience or building blocks, circuits design via NAM expansion is a systematic methodology with no need to consider the

\footnotetext{
* Correspondence: yugz@buaa.edu.cn

School of Transportation Science and Engineering, Beihang University, 100191 Beijing, China
}

final topology of the obtained circuits during the process of design [7]. So, the method of NAM expansion provides a bridge between the practical circuit design and theory. And, it is already applied to filter [8] and oscillator [9] design.

Circuit design using NAM expansion is a novel subject in theory of active network synthesis. As OPA is prevalent devices in circuit, this paper firstly provides generation method of the OPA-based TowThomas (TT) Bi-quad band-pass filter circuit using NAM expansion, in which the generation process is based on a symbolic method of circuit design, and the derived circuit originates from a symbolic transfer function. As extra nullors are introduced, nullators and norators may be paired as operational amplifiers in alternative ways, then different active filter circuits can be derived from the same circuit network with nullors. Thus, the Tow-Thomas band-stop filter circuit and Åkerberg-Mossberg band-stop filter circuit are taken as examples to illustrate the synthesis method by nodal admittance matrix expansion on the same port admittance matrix.

\section{Theory of NAM expansion}

2.1 Nullor and active device modeling

The pathological elements of nullor, that are the nullator and the norator, are specified according to the constraints they impose on their terminal voltage and current. For the nullator, shown in Fig. $1 \mathrm{~b}, v=i=0$, 


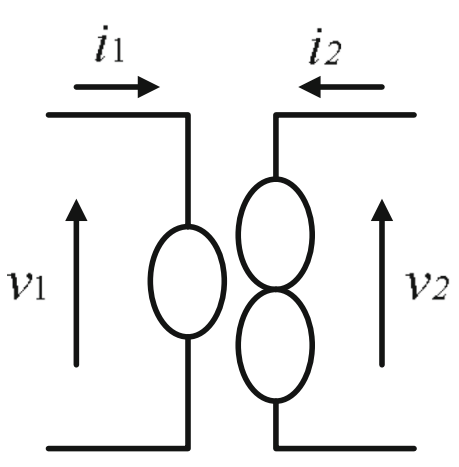

(a)

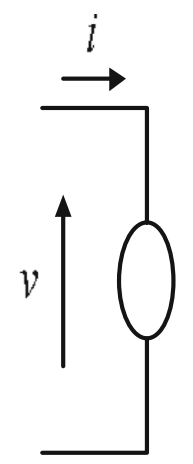

(b)

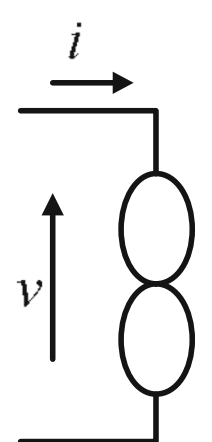

(c)

Fig. 1 a-c Nullor equivalence

which enables it to be used for non-inverting voltage conveying, while for the norator, shown in Fig. 1c, it imposes no constraints on its terminal voltage and the current flowing into is equal to the current flowing out, which enables it to be used for noninverting current conveying [10].

Nullors can be used to model various controlled sources as well as varieties of ideal active devices, such as OPA and the second generation current conveyor (CCII-). Nullor equivalences of the OPA and the CCIIare shown in Fig. 2.

\subsection{Introduction of nullors into NAM}

For a NAM with $N$ nodes, the same rows and columns with zero terms can be added to the original matrix. Considering the row $n$ is full of zero terms and node $n$ is an internal node within the network, a norator can be connected between node $n$ and an arbitrary node $m$ (including the port node and the reference node), for input current $I n=0$. While considering the column $k$ is full of zero terms and node $k$ is an internal node within the network, a nullator can be connected between node $k$ and an arbitrary node $j$ (including the port node and the reference node), which made the voltage of $k$ equal to the voltage of node $j$. Process of introduction can be illustrated in Eq. (1).

$$
\left[\begin{array}{cccccccc}
y_{11} & y_{12} & \cdots & y_{1 j} & \cdots & y_{1 k} & \cdots & y_{1 N} \\
y_{21} & y_{22} & \cdots & y_{2 j} & \cdots & y_{2 k} & \cdots & y_{2 N} \\
\vdots & \vdots & \cdots & \vdots & \cdots & \vdots & \cdots & \vdots \\
y_{m 1} & y_{m 2} & \cdots & y_{m j} & \cdots & y_{m k} & \cdots & y_{m N} \\
\vdots & \vdots & \cdots & \vdots & \cdots & \vdots & \cdots & \vdots \\
y_{n 1} & y_{n 2} & \cdots & y_{n j} & \cdots & y_{n k} & \cdots & y_{n N} \\
\vdots & \vdots & \cdots & \vdots & \cdots & \vdots & \cdots & \vdots \\
y_{N 1} & y_{N 2} & \cdots & y_{N j} & \cdots & y_{N k} & \cdots & y_{N N}
\end{array}\right]
$$

For the convenience of matrix processing, the variable- $\infty$ proposed in reference [11] has been introduced to represent the connected nullor.

$$
\left[\begin{array}{cclccccc}
y 11 & y_{12} & \cdots & y_{1 j} & \cdots & y_{1 k} & \cdots & y_{1 N} \\
y 21 & y_{22} & \cdots & y_{2 j} & \cdots & y_{2 k} & \cdots & y_{2 N} \\
\vdots & \vdots & \cdots & \vdots & \cdots & \vdots & \cdots & \vdots \\
y_{m 1} & y_{m 2} & \cdots & y_{m j}+\infty_{i} & \cdots & y_{m k}-\infty_{i} & \cdots & y_{m N} \\
\vdots & \vdots & \cdots & \vdots & \cdots & \vdots & \cdots & \vdots \\
y_{n 1} & y_{n 2} & \cdots & y_{n j}-\infty_{i} & \cdots & y_{n k}+\infty_{i} & \cdots & y_{n N} \\
\vdots & \vdots & \cdots & \vdots & \cdots & \vdots & \cdots & \vdots \\
y_{N 1} & y_{N 2} & \cdots & y_{N j} & \cdots & y_{N k} & \cdots & y_{N N}
\end{array}\right]
$$

In Eq. (2), variable $i$ indicates the $i$ th nullor, $\infty_{i}$ is a linked infinity parameter. During matrix manipulating, terms in columns $j$ and $k$ connected between a nullator

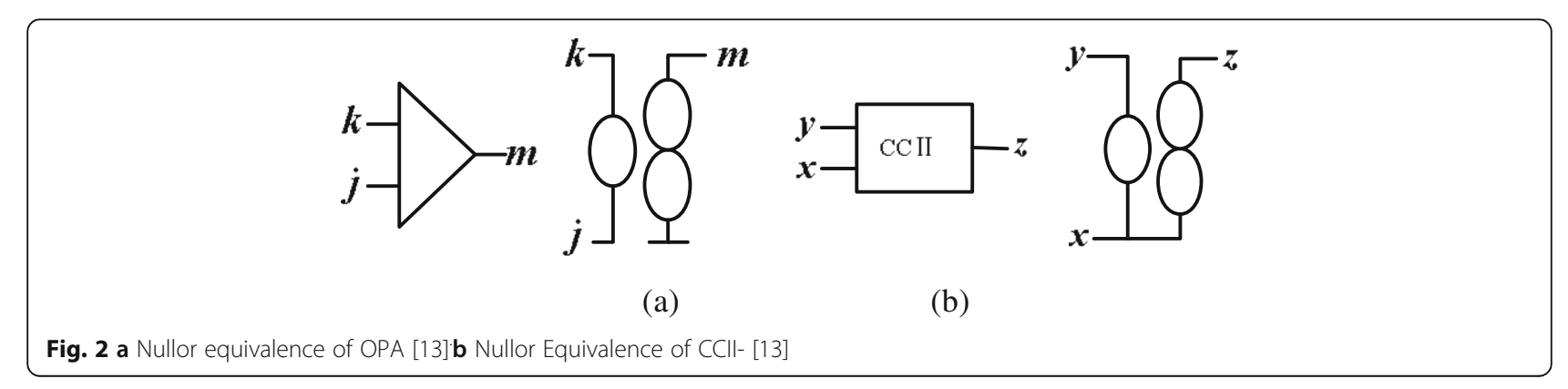


Table 1 Port admittance matrix descriptions of VCVS and CCCS [12]

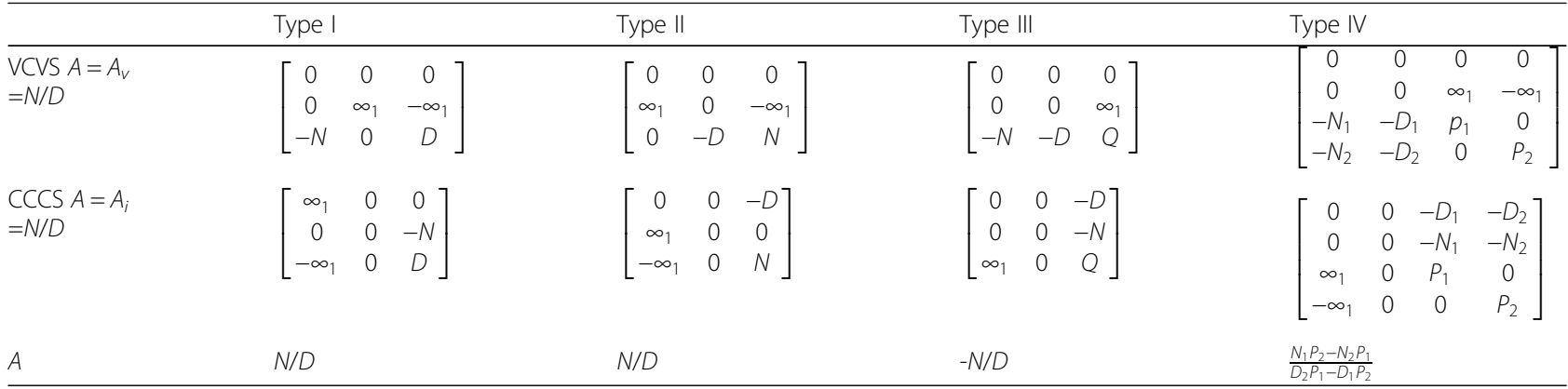

can be moved between the two columns without affecting the equivalent circuit characteristics, while terms in rows $m$ and $n$ connected between a norator can be moved between the two rows. Term moving is called the element shift theorem. In particular, if there is $\infty_{i}$ existing at the matrix, any terms can be added to the row and the column where $\infty_{i}$ exists, while if there is $\pm \infty_{i}$ existing at the same row of the matrix, then any terms can be added to the row, and if there is $\pm \infty_{i}$ existing at the same columns, then any terms can be added to the column [12]. Term adding is called the arbitrary element theorem.

\subsection{Theory of pivotal expansion and Gaussian elimination} In an ideal transistor and OPA active circuit, passive linear elements can be described by a symmetric NAM, and the active elements can be described by nullors. If element $t+r q / s$ is regarded as the pivotal element, then the matrix after pivotal expansion is equivalent to the original 1-port matrix, in which the signs are selected that the number of minus signs is odd [12].

$$
\left[t+\frac{r q}{s}\right] \rightarrow\left[\begin{array}{cc}
t+\frac{r q}{s} & 0 \\
0 & 0
\end{array}\right] \rightarrow\left[\begin{array}{cc}
t & \mp q \\
\pm r & \pm s
\end{array}\right]
$$

\subsection{Admittance matrix descriptions for circuits with prescribed voltage and current transfer functions}

Circuit analysis consists of solving the transfer function of a circuit, such as the open-circuit voltage gain and the short-circuit current gain, through which the performance of the circuit can be analyzed. Circuit synthesis happens to be the inverse process of the circuit analysis. That is, given the transfer function $A_{v}$ or $A_{i}$, starting from the NAM of the voltage controlled voltage source (VCVS) or the current controlled current source (CCCS) shown in Table 1, the circuit satisfying the requirements can be obtained. In Table $1, N$ and $D$ respectively represent the numerator and denominator of the transfer function, $Q$ represents an arbitrary admittance function parameter.

\section{The method of active network synthesis}

Just like passive filter synthesis, different orders of removing transmission zero result in different circuit topology. Different form of transfer function may lead to different structure of active filter. The process of passive-RC circuit synthesis consists of the following six steps [3]:

1 Choose a suitable matrix from Table 1 according to the given transfer function.

2 Determine each element of the starting matrix referring to the transfer function.

3 Carry out expansion of $N, D, P$, or $Q$ terms until all terms become 1st-order admittance functions.

4 Introducing missing terms or shift original matrix terms according to the arbitrary element theorem and element shift theorem proposed in Section 2.2.

5 If all matrix elements now correctly describe passive elements, the circuit is a single nullor circuit.

6 Otherwise, add extra nullors to introduce missing matrix terms.

\section{Active filter synthesis using nodal admittance matrix expansion}

4.1 Active band-pass filter synthesis

Assuming the voltage transfer function is given as

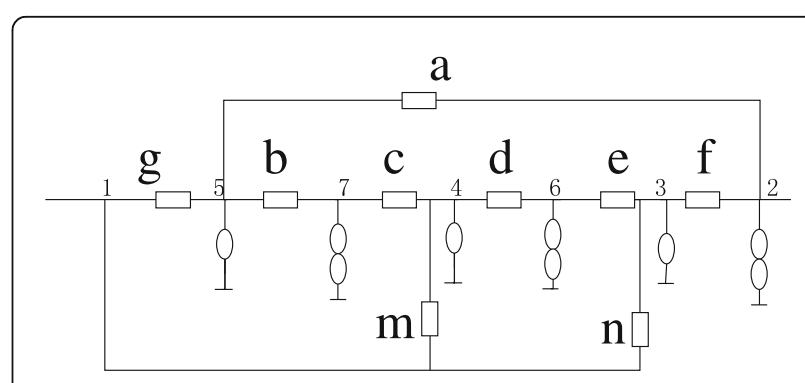

Fig. 3 Synthesized TT Bi-quad circuit 


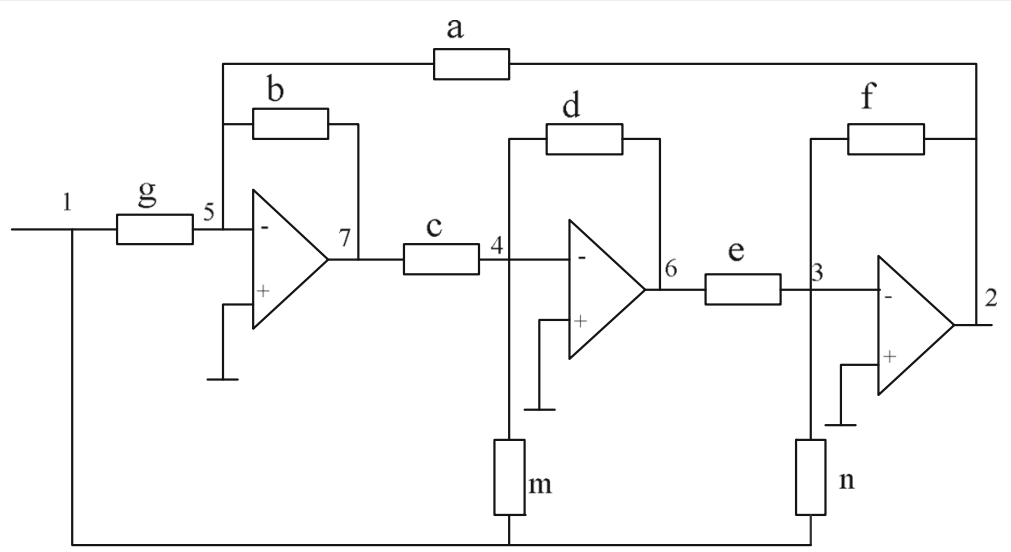

Fig. 4 OPA realization of $\Pi$ Bi-quad circuit

$$
A v=-\frac{c e g-b e m+b d n}{a c e+b d f}
$$

According to the extended form of the voltage and current transfer functions provided in Table 1, the type III of the VCVS is selected. That is,

$\left[\begin{array}{ccc}0 & 0 & 0 \\ 0 & 0 & \infty_{1} \\ -N & -D & Q\end{array}\right] \rightarrow\left[\begin{array}{ccc}0 & 0 & 0 \\ 0 & 0 & \infty_{1} \\ \frac{- \text { ceg }+ \text { bem-bdn }}{Q_{1}} & -\frac{\text { ace }+ \text { bdf }}{Q_{1}} & Q\end{array}\right]$

The first-order admittance function of $Q_{1}$ has been introduced in Eq. (5). Appropriate selection of $Q_{1}$ makes the expansion process more efficient. Then, $Q_{1}$ is selected to equal to bd.

$$
\left[\begin{array}{lrr}
0 & 0 & 0 \\
0 & 0 & \infty_{1} \\
-n+\frac{\mathrm{em}}{d}-\frac{\mathrm{ceg}}{\mathrm{bd}}-f-\frac{\mathrm{ace}}{\mathrm{bd}} & Q
\end{array}\right]
$$

After applying the pivotal expansion to the elements of the $-n+\frac{\mathrm{em}}{d}-\frac{\mathrm{ceg}}{\mathrm{bd}}$ and $-f-\frac{\mathrm{ace}}{\mathrm{bd}}$, Eq. (7) is obtained, where pivotal expansion is implemented twice.

$$
\left[\begin{array}{lllrr}
0 & 0 & 0 & 0 & 0 \\
0 & 0 & \infty_{1} & 0 & 0 \\
-n & -f & Q & -e & 0 \\
-m & 0 & 0 & -d & -c \\
-g & -a & 0 & 0 & -b
\end{array}\right]
$$

For any terms can be added to the second row and the third column, Eq. (7) can be transformed to Eq. (8), where there is an element of $f$ in $Q$. As a floating element, $f$ is connected between node 2 and node 3 . Viewing that the other elements can be described in the same manner as $f$, two columns of zero terms were

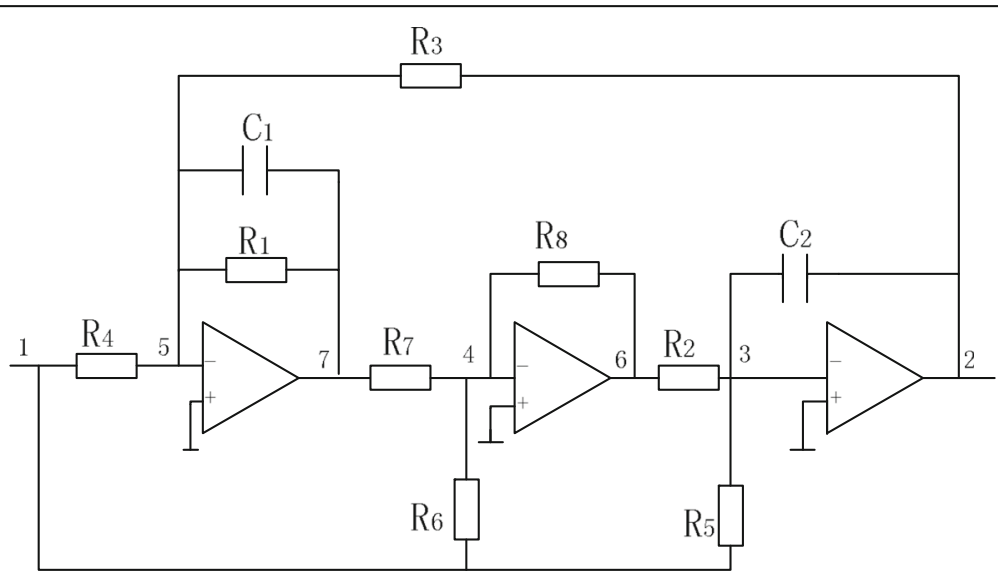

Fig. 5 Tा Bi-quad circuit with forward feedback 
Table 2 Component values of the designed band-pass filter $C_{1}=C_{2}=1 \mathrm{nF} \quad R_{1}=R_{2}=R_{3}=R_{4}=R_{5}=R_{7}=R_{8}=15.915 \mathrm{k} \Omega \quad R_{6}=7.957 \mathrm{~K} \Omega$

added next to the third column, two rows of zero terms were added under the fifth row. Then Eq. (9) equivalent to Eq. (8) is obtained.

$$
\begin{aligned}
& {\left[\begin{array}{lllll}
0 & 0 & 0 & 0 & 0 \\
0 & f & -f+\infty_{1} & 0 & 0 \\
-n & -f & Q & -e & 0 \\
-m & 0 & 0 & -d & -c \\
-g & -a & 0 & 0 & -b
\end{array}\right]} \\
& {\left[\begin{array}{lllrlrr}
0 & 0 & 0 & 0 & 0 & 0 & 0 \\
0 & f & -f+\infty_{1} & 0 & 0 & 0 & 0 \\
-n & -f & Q & 0 & 0 & -e & 0 \\
-m & 0 & 0 & 0 & 0 & -d & -c \\
-g & -a & 0 & 0 & 0 & 0 & -b \\
0 & 0 & 0 & 0 & 0 & 0 & 0 \\
0 & 0 & 0 & 0 & 0 & 0 & 0
\end{array}\right]}
\end{aligned}
$$

Infinity variables $\infty_{2}$ and $\infty_{3}$ are introduced to the newly added rows and columns, then corresponding terms are added to make elements appear in the form of floating or grounding.

$$
\left[\begin{array}{lllllll}
0 & 0 & 0 & 0 & 0 & 0 & 0 \\
0 & a+f & -f+\infty_{1} & 0 & -a & 0 & 0 \\
-n & -f & Q & 0 & 0 & -e & 0 \\
-m & 0 & 0 & c+d & 0 & -d & -c \\
-g & -a & 0 & 0 & a+b & 0 & -b \\
0 & 0 & -e & -d+\infty_{2} & 0 & d+e & 0 \\
0 & 0 & 0 & -c & -b+\infty_{3} & 0 & b+c
\end{array}\right]
$$

The position of the introduced nullors indicates that the norator and nullator of the second nullor are connected respectively between node 6 and the reference node, node 4 and the reference node, while the norator and nullator of the third nullor are connected respectively between node 7 and the reference node, node 5 and the reference node. The admittance function $Q$ contains a term of $e$.

The terms $m,-m$ and $n,-n$ are moved to the first row from node 0 . The admittance function $Q$ equals to $e+f+n$.

$\left[\begin{array}{lllllll}g+m+n & 0 & -n & -m & -g & 0 & 0 \\ 0 & a+f & -f+\infty_{1} & 0 & -a & 0 & 0 \\ -n & -f & e+f+n & 0 & 0 & -e & 0 \\ -m & 0 & 0 & m+c+d & 0 & -d & -c \\ -g & -a & 0 & 0 & g+a+b & 0 & -b \\ 0 & 0 & -e & -d+\infty_{2} & 0 & d+e & 0 \\ 0 & 0 & 0 & -c & -b+\infty_{3} & 0 & b+c\end{array}\right]$

Then, Eq. (11) obtained described the TT Bi-quad circuit in Fig. 3.

Obviously, Fig. 3 can be realized by OPA combined with passive elements. The nullors in Fig. 3 can be respectively replaced by nullor equivalence of the OPA, as shown in Fig. 4.

For realization, $a$ is selected as a resistor $g_{3}, b$ is selected as a resistor combined with a capacitor, that is $C_{1} s+g_{1}, c$ is selected as a resistor $g_{7}, d$ is selected as a resistor $g_{8}, e$ is selected as a resistor $g_{2}, f$ is selected as a capacitor, that is $C_{2} s, g$ is selected as a resistor $g_{4}, m$ is selected as a resistor $g_{6}, n$ is selected as a resistor $g_{5}$, then Eq. (4) yields

$$
A_{v}=-\frac{g_{5} g_{8}\left(C_{1} s+g_{1}\right)+g_{2} g_{4} g_{7}-g_{2} g_{6}\left(C_{1} s+g_{1}\right)}{g_{8} C_{2} s\left(C_{1} s+g_{1}\right)+g_{2} g_{3} g_{7}}
$$

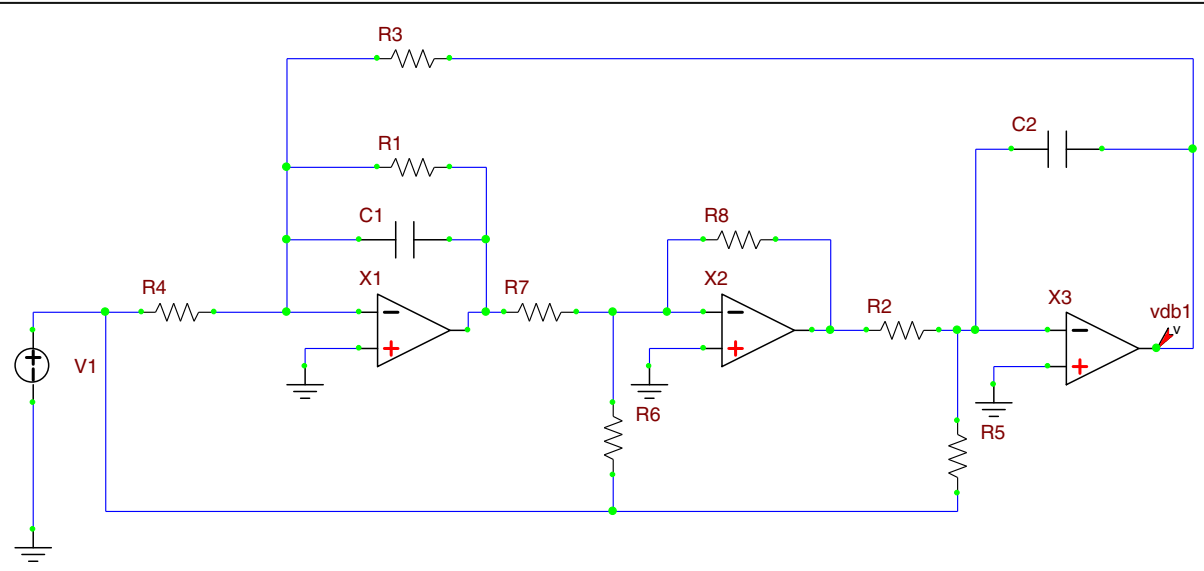

Fig. 6 The simulation of $\Pi$ Bi-quad band-pass filter 


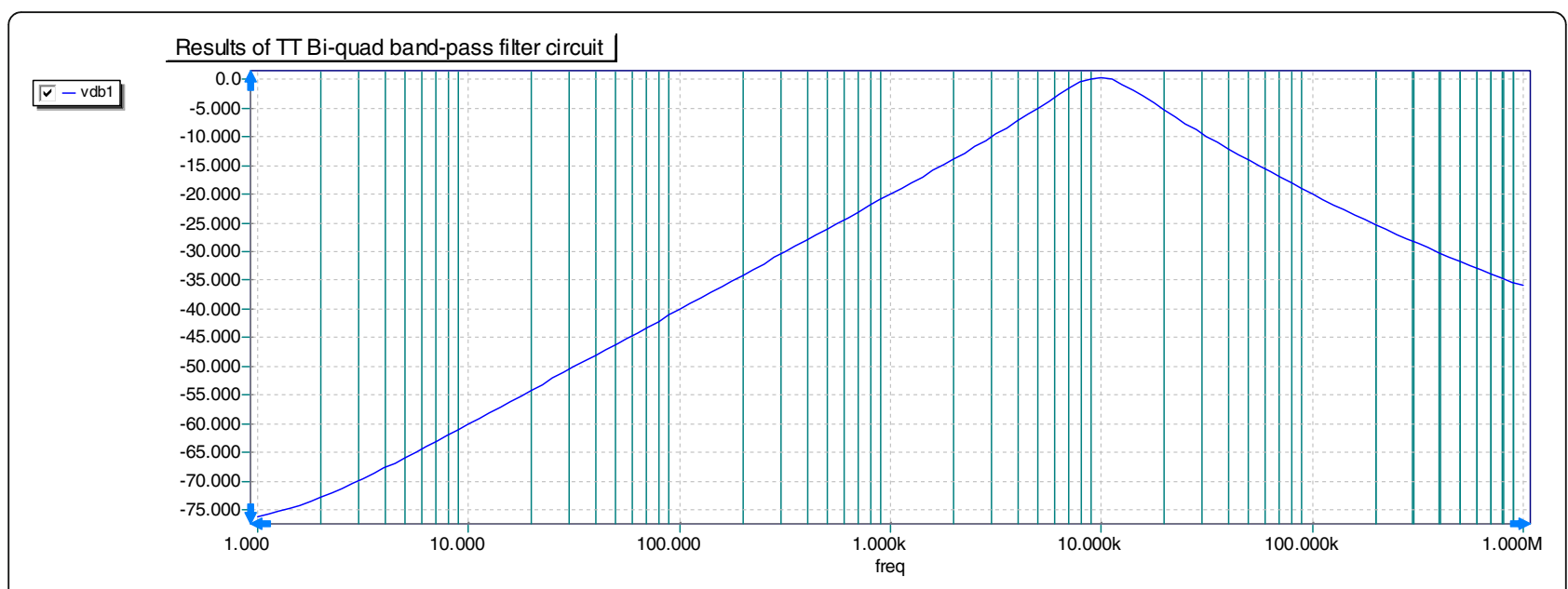

Fig. 7 The simulation results of $\Pi \mathrm{B}$ Bi-quad band-pass filter

$$
\begin{gathered}
A_{v}=-\frac{g_{2} g_{4} g_{7}+g_{1} g_{5} g_{8}-g_{1} g_{2} g_{6}+s C_{1}\left(g_{5} g_{8}-g_{2} g_{6}\right)}{g_{8} C_{1} C_{2} s^{2}+g_{1} g_{8} C_{2} s+g_{2} g_{3} g_{7}} \\
A_{v}=-\frac{g_{2} g_{4} \frac{g_{7}}{g_{8}}+g_{1} g_{5}-g_{1} g_{2} \frac{g_{6}}{g_{8}}+s C_{1}\left(g_{5}-g_{2} \frac{g_{6}}{g_{8}}\right)}{C_{1} C_{2} s^{2}+g_{1} C_{2} s+g_{2} g_{3} \frac{g_{7}}{g_{8}}}
\end{gathered}
$$

Then, the circuit of Fig. 5 is obtained as the TT Biquad circuit with forward feedback.

A design example is provided to illustrate the design procedure. Design a band-pass filter according to the parameters below:

$$
\mathrm{fp}=10 \mathrm{kHz}, \mathrm{Qp}=1, K=1
$$

where fp represents the pass-band central frequency, Qp represents the quality factor, $K$ represents the stop-band voltage magnification.

$$
A_{\mathrm{v}}(\mathrm{s})=\frac{K \frac{\omega_{p}}{Q_{p}} s}{s^{2}+\frac{\omega_{p}}{Q_{p}} s+\omega_{p}^{2}}
$$

In this design example, for simplicity, capacitor is chosen to $1 \mathrm{nF}$. Component values which are calculated according to Eq. (15) are listed in Table 2.

\subsection{Active band-stop filter synthesis}

The transfer function of a band-stop filter may be in the form the TT Bi-quad circuit is simulated according to the component values presented above respectively, which are shown in Figs. 6 and 7

$$
A_{v}=-\frac{k\left(s^{2}+\omega_{z}^{2}\right)}{s^{2}+\frac{\omega_{0}}{Q_{0}} s+\omega_{0}^{2}}
$$

Assuming the elements $C_{1}, C_{2}, C_{3}, g_{1}, g_{2}, g_{3}, g_{4}, g_{5}$, and $g_{6}$ are used to build the band-stop filter (Fig. 6), where $g_{1}$ is used for tuning of $\omega_{\mathrm{z}}, g_{2}, g_{3}$, and $g_{4}$ may be used to form the gain of the amplifier, $g_{6}$ is used for tuning of $\omega_{0}, g_{5}$ is used for tuning of $Q_{0}$. The transfer function is then in the form

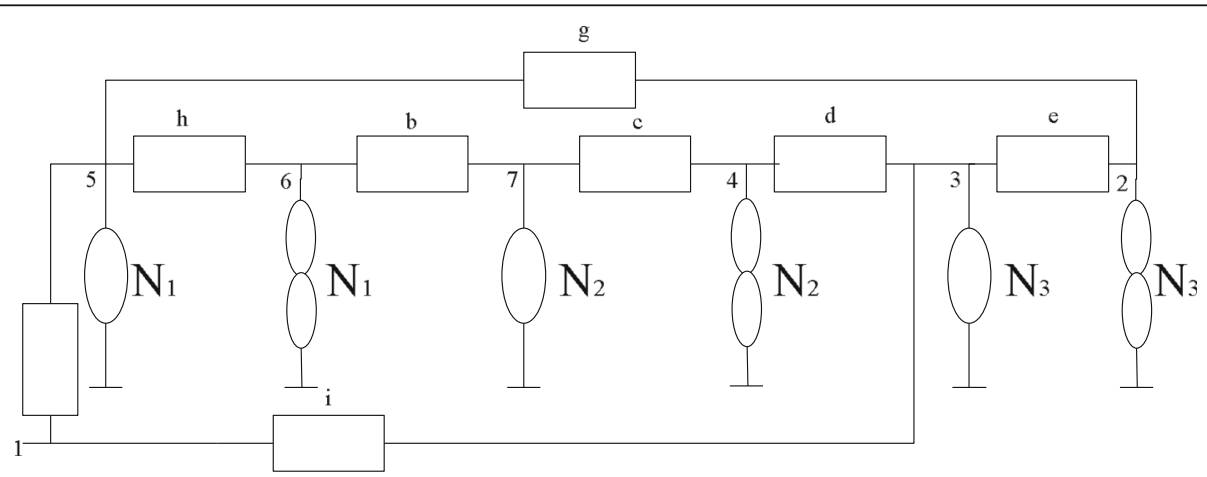

Fig. 8 The circuit topology synthesized from Eq. (18) 


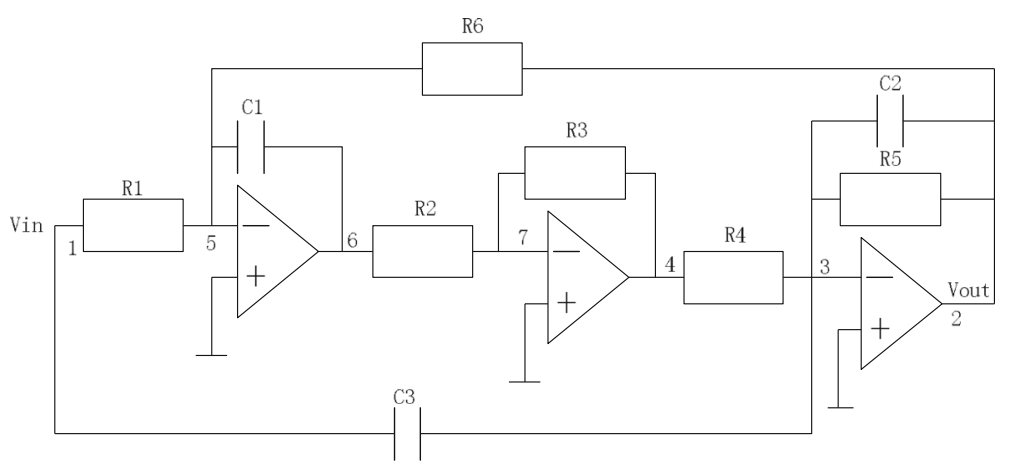

Fig. 9 Tow-Thomas band-stop filter circuit

$$
\begin{aligned}
A_{v} & =-\frac{C_{1} C_{3} s^{2}+g_{1} g_{4} \frac{g_{2}}{g_{3}}}{C_{1} C_{2} s^{2}+C_{1} g_{5} s+g_{4} g_{6} \frac{g_{2}}{g_{3}}} \\
& =-\frac{g_{3} C_{1} C_{3} s^{2}+g_{1} g_{4} g_{2}}{g_{3} C_{1} C_{2} s^{2}+g_{3} C_{1} g_{5} s+g_{4} g_{6} g_{2}} \\
& =-\frac{g_{3} C_{1} C_{3} s^{2}+g_{1} g_{4} g_{2}}{g_{3} C_{1} s\left(C_{2} s+g_{5}\right)+g_{4} g_{6} g_{2}}
\end{aligned}
$$

For simplicity, let $a$ stand for $g_{1}, b$ stand for $g_{2}, c$ stand for $g_{3}, d$ stand for $g_{4}, e$ stand for $C_{2} s+g_{5}, g$ stand for $g_{6}$, $h$ stand for $C_{1} s, i$ stand for $C_{3} s$, yields

$$
A_{v}=-\frac{\text { ihc }+ \text { abd }}{\text { ehc }+ \text { gbd }}
$$

From the given transfer function, type III in Table 1 is selected according to the content description in Section 2.4 , that is,

$$
A_{v}=-\frac{N}{D}
$$

Allocate every terms of the matrix after choosing transfer function

$$
\begin{aligned}
& {\left[\begin{array}{ccc}
0 & 0 & 0 \\
0 & 0 & \infty_{1} \\
-N & -D & Q
\end{array}\right] \stackrel{\begin{array}{c}
N=\mathrm{ihc}+\mathrm{abd} \\
D=\mathrm{ehc}+\mathrm{gbd} \\
Q=(d+e+\mathrm{i} h \mathrm{hc}
\end{array}}{\longrightarrow}} \\
& {\left[\begin{array}{ccc}
0 & 0 & 0 \\
0 & 0 & \infty_{1} \\
-\frac{\mathrm{ihc}+\mathrm{abd}}{Q_{2}} & -\frac{\text { ehc }+\mathrm{gbd}}{Q_{2}} & \frac{(d+e+i) \mathrm{hc}}{Q_{2}}
\end{array}\right]}
\end{aligned}
$$

An arbitrary 1st-order function $Q_{2}$ is introduced to Eq. (20) in order to carry out pivotal expansion, where $Q_{2}=$ hc. After pivotal expansion, another function $Q_{3}=b$ is introduced to the right side matrix of Eq. (21).

$$
\begin{aligned}
& {\left[\begin{array}{ccc}
0 & 0 & 0 \\
0 & 0 & \infty_{1} \\
-i-\frac{a b d}{h c} & -e-\frac{g b d}{h c} & d+e+i
\end{array}\right] \rightarrow} \\
& {\left[\begin{array}{cccc}
0 & 0 & 0 & 0 \\
0 & 0 & \infty_{1} & 0 \\
-i & -e & d+e+i & -d \\
\hdashline-\frac{a b}{Q_{3}} & -\frac{g b}{Q_{3}} & 0 & \frac{h c}{Q_{3}}
\end{array}\right]}
\end{aligned}
$$

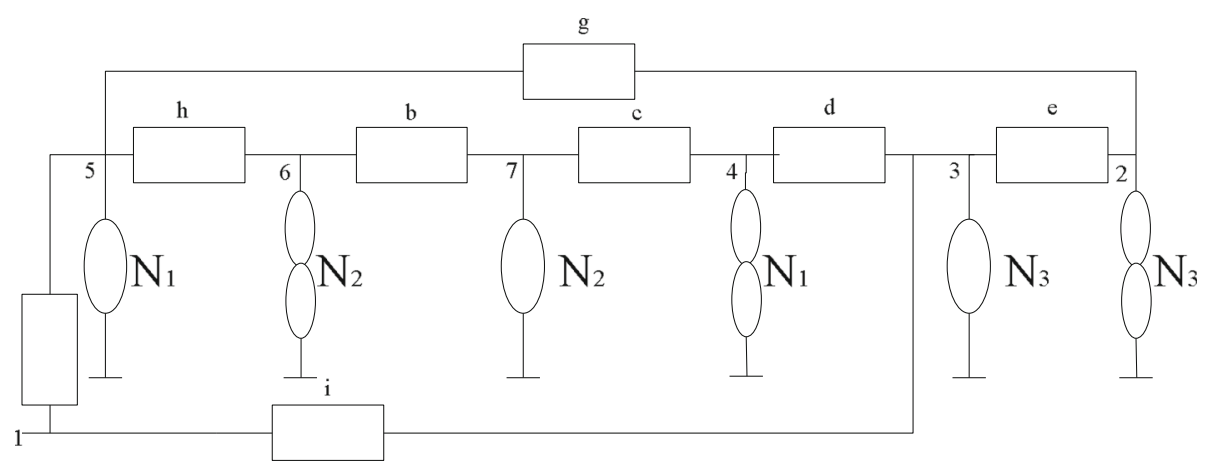

Fig. 10 The reconstructed circuit topology 


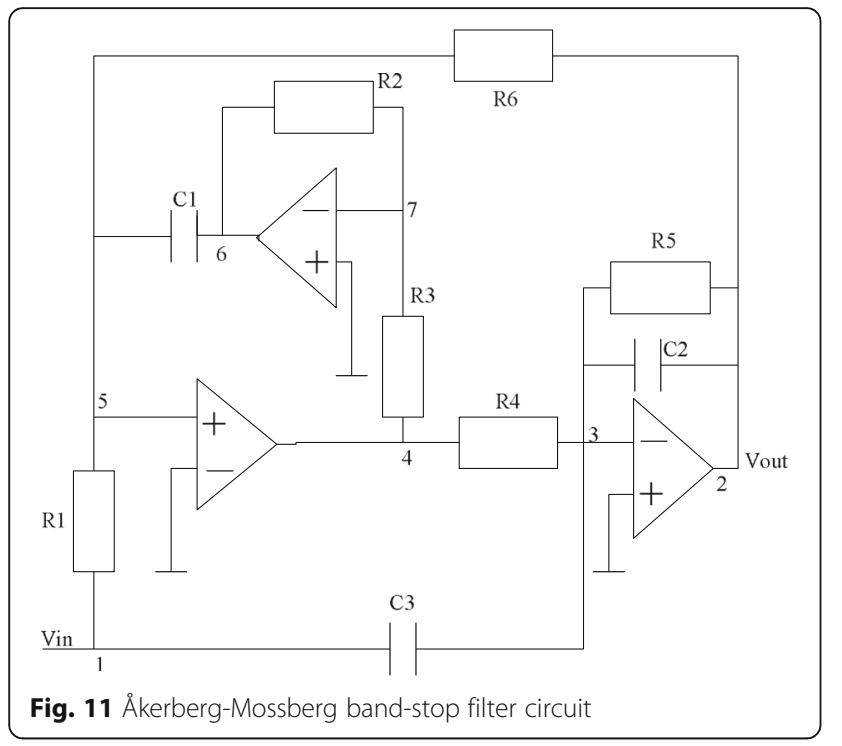

Carry pivotal expansion on the terms in the fourth row of the right side matrix of Eq. (21), we get Eq. (22) (Fig. 7).

$$
\begin{aligned}
& {\left[\begin{array}{ccc:c:c}
0 & 0 & 0 & 0 \\
0 & 0 & \infty_{1} & 0 \\
-i & -e & d+e+i & -d \\
\hdashline-a & -g & 0 & \frac{h c}{b}
\end{array}\right] \rightarrow} \\
& {\left[\begin{array}{cccc:c}
0 & 0 & 0 & 0 & 0 \\
0 & 0 & \infty_{1} & 0 & 0 \\
-i & -e & d+e+i & -d & 0 \\
-a & -g & 0 & 0 & -h \\
\hdashline 0 & 0 & 0 & -c & -b
\end{array}\right]}
\end{aligned}
$$

According to the arbitrary element theorem, $\pm e$ elements are introduced to the row where $\infty_{1}$ exists. The $\pm i$ elements are moved to the first row from the zeroth
Table 3 Component values of the designed band-stop filter

\begin{tabular}{lll}
\hline$C_{1}=3.17 \mathrm{nF}$ & $R_{1}=R_{2}=R_{3}=R_{4}=1 \mathrm{k} \Omega$ & $R_{5}=159.15 \Omega$ \\
\hline$C_{2}=1 \mathrm{uF}$ & $C_{3}=2 \mathrm{uF}$ & $R_{6}=2 \mathrm{~K} \Omega$ \\
\hline
\end{tabular}

row. Then, the equivalent matrix of Eq. (22) is obtained as Eq. (23).

$$
\left[\begin{array}{cccc:c}
i & 0 & -i & 0 & 0 \\
0 & e & \infty_{1}-e & 0 & 0 \\
-i & -e & d+e+i & -d & 0 \\
-a & -g & 0 & 0 & -h \\
\hdashline 0 & 0 & 0 & -c & -b
\end{array}\right]
$$

Extra nullors need to be introduced since the elements $-a,-b,-c,-d,-h$, and $-g$ cannot be represented by floating terms. The matrix of Eq. (24) can be obtained by introducing all zeroes in the 4th and 6th rows and all zeroes in the 5th and 7th columns; adding a nullator between node 5 and the grounded node, and another one between node 7 and the grounded node; adding a norator between node 4 and the grounded node and another one between node 6 and the grounded node.

$$
\left[\begin{array}{ccccccc}
i & 0 & -i & 0 & 0 & 0 & 0 \\
0 & e & \infty_{1}-e & 0 & 0 & 0 & 0 \\
-i & -e & d+e+i & -d & 0 & 0 & 0 \\
0 & 0 & 0 & 0 & \infty_{3} & 0 & 0 \\
-a & -g & 0 & 0 & 0 & -h & 0 \\
0 & 0 & 0 & 0 & 0 & 0 & \infty_{2} \\
0 & 0 & 0 & -c & 0 & -b & 0
\end{array}\right]
$$

According to the arbitrary element theorem, Eq. (25) is obtained.

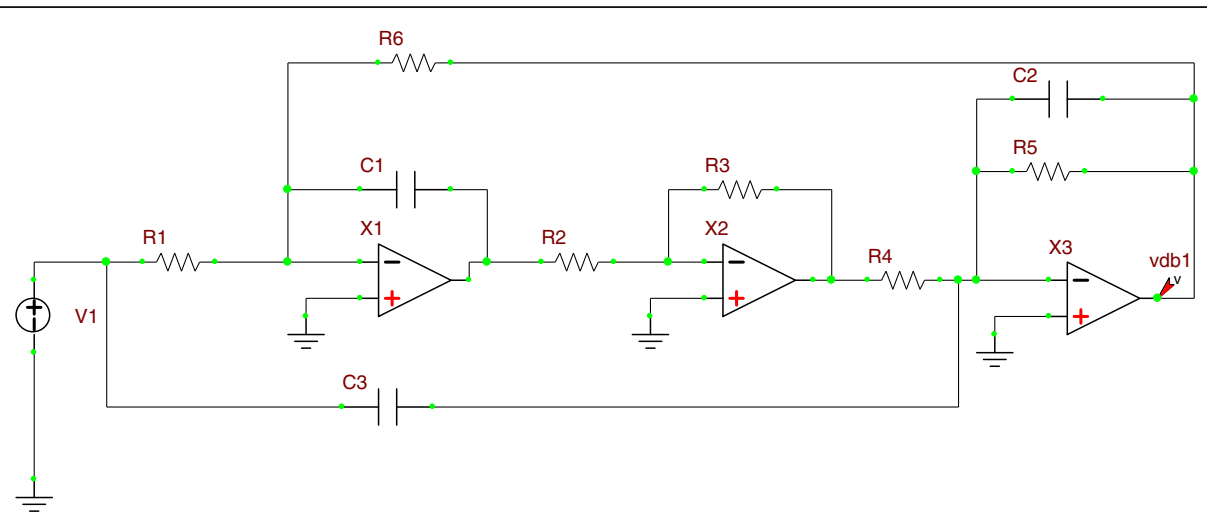

Fig. 12 The simulation of $\pi$ band-stop filter 


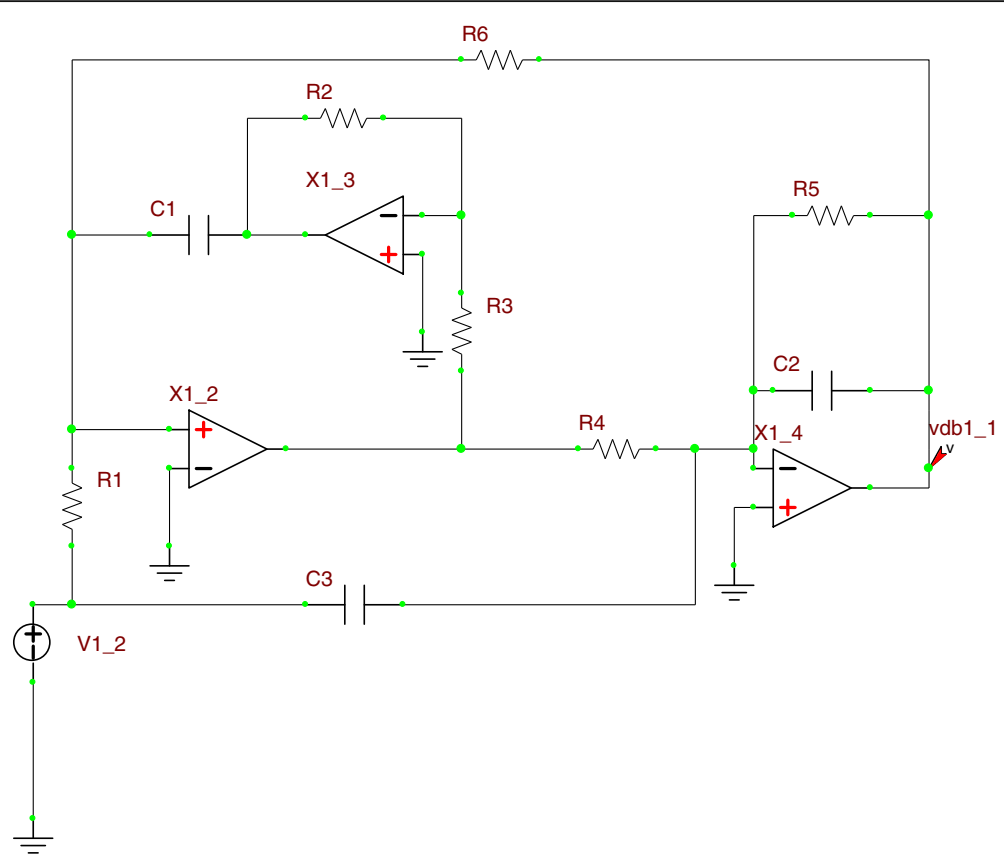

Fig. 13 The simulation of Åkerberg-Mossberg band-stop filter

$\left[\begin{array}{ccccccc}a+i & 0 & -i & 0 & -a & 0 & 0 \\ 0 & e+g & \infty_{1}-e & 0 & -g & 0 & 0 \\ -i & -e & d+e+i & -d & 0 & 0 & 0 \\ 0 & 0 & -d & c+d & \infty_{3} & 0 & -c \\ -a & -g & 0 & 0 & a+g+h & -h & 0 \\ 0 & 0 & 0 & 0 & -h & b+h & \infty_{2}-b \\ 0 & 0 & 0 & -c & 0 & -b & b+c\end{array}\right]$

It can be seen that the circuit includes three pairs of nullors from Eq. (25). For one pair, the nullator is connected between node 3 and the grounded node, and the norator between node 2 and the grounded node. For another pair, the nullator is connected between node 5 and the grounded node, and the norator between node 6 and the grounded node. And for the third one, the nullator is connected between node 7 and the grounded node, and the norator between node 4 and the grounded node. Therefore, the new active circuit topology is obtained as shown in Fig. 8.
As assumed before, the elements $a, b, c, d$, and $g$ are selected as the resistor; $h$ and $i$ as the capacitor; $e$ as the combination of a resistor and a capacitor; and nullors are in pair in succession from the input node to the output node. Then, the Tow-Thomas band-stop filter circuit shown in Fig. 9 is obtained.

Alternatively, if we reorder the nullors in Fig. 8 as the following sequence shown in Fig. 10, then the reconstructed circuit topology with nullors replaced by OPA is synthesized to the Åkerberg-Mossberg band-stop filter, which is shown in Fig. 11.

Band-stop filter can be obtained by Eq. (26), and a design example is provided to illustrate the design procedure. Design a band-stop filter according to the parameters below:

$$
f p=2 \mathrm{kHz}, Q p=2, K=2
$$

Where fp represents the stop-band central frequency, Qp represents the quality factor, $K$ represents the passband voltage magnification.

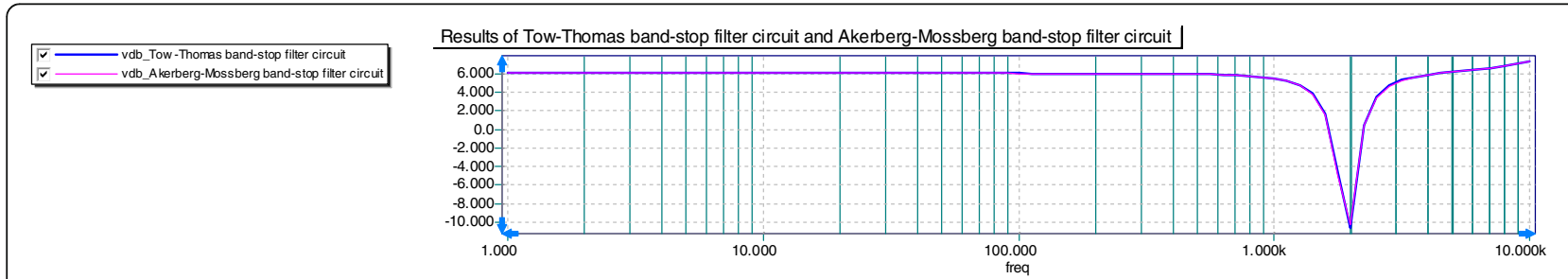

Fig. 14 The simulation results of $\Pi$ band-stop filter circuit and Åkerberg-Mossberg band-stop filter circuit 


$$
A_{V}(S)=\frac{K\left(S^{2}+\omega_{p}{ }^{2}\right)}{S^{2}+\frac{\omega_{p}}{Q_{p}} S+\omega_{p}{ }^{2}}
$$

In this design example, for simplicity, capacitor $C_{2}$ is chosen to $1 \mathrm{uF}$, and $R_{1}=R_{2}=R_{3}=R_{4}=1 \mathrm{k} \Omega$. Component values which are calculated according to Eq. (26) are listed in Table 3.

The TT band-stop filter and the Åkerberg-Mossberg band-stop filter are simulated according to the component values presented above respectively, which are shown in Figs. 12 and 13.

The simulation results are shown in Fig. 14, which explains that the results of the two band-stop filter are consistent.

\section{Conclusions}

The generation method of the TT Bi-quad band-pass circuit, the TT band-stop filter circuit, and ÅkerbergMossberg band-stop filter circuit is presented using the theory of NAM expansion, which is a new design method for circuit design. The active circuit topologies of the TT band-stop filter and the Åkerberg-Mossberg band-stop filter are synthesized from the same transfer function. The analysis in the paper verifies the effectiveness of the theory of NAM expansion for circuit design in theory and practice. At the same time, further research needs to be conducted to enrich the method of NAM expansion.

\section{Acknowledgements}

There is no other one to acknowledge in this section.

\section{Funding}

This research was funded partially by the National Science Foundation of China under grant no. 61371076.

\section{Competing interests}

The authors declare that they have no competing interests.

\section{Authors' contributions}

LT as the first author wrote the manuscript and carried out all the simulation of examples in this manuscript. YW as the supervisor of Lingling Tan provided some guidance on the method of circuit design base on nodal admittance matrix expansion. GY as the corresponding author provided some suggestions on the English writing. All authors read and approved the final manuscript.

\section{Publisher's Note}

Springer Nature remains neutral with regard to jurisdictional claims in published maps and institutional affiliations.

Received: 6 April 2017 Accepted: 10 May 2017

Published online: 26 May 2017

\section{References}

1. T Yanagisawa, N Kambayashi, Realization of arbitrary conductance matrix using operational amplifiers. IEICE Trans. Part A J59-A(5), 401-408 (1976)

2. DG Haigh, P Radmore et al., Systematic synthesis method for analogue circuits.Part I. Notation and synthesis toolbox. ISCAS I, 701-704 (2004)
3. DG Haigh, A method of transformation from symbolic transfer function to active-RC circuit by admittance matrix expansion. IEEE Trans. Circuits Syst. I, Reg. Papers 53(12), 2715-2728 (2006)

4. RA Saad, AM Soliman, Generation, modeling, and analysis of CCII-based gyrators using the generalized symbolic framework for linear active circuits. International Journal of Circuit Theory and Applications 36(3), 289-309 (2008)

5. IA Awad, AM Soliman, On the voltage mirrors and the current mirrors. Analog Integrated Circuits and Signal Processing 32(1), 79-81 (2002)

6. RA Saad, AM Soliman, Use of mirror elements in the active device synthesis by admittance matrix expansion. IEEE Trans. Circuits Syst. I 55, 2726-2735 (2008)

7. Lingling Tan, Yu Bai, Jianfu Teng, Kaihua Liu, Wenqing Meng, Transimpedance filter synthesis based on nodal admittance matrix expansion, circuits, Systems \&amp; Signal Processing. (2012). dio:10.1007/s00034-0129514-y

8. AM Soliman, Two integrator loop filters: generation using NAM expansion and review. J. Electr. Comput. Eng 108687(2010), 2010 (2010). doi:10.1155/ 2010/108687

9. AM Soliman, Generation of current conveyor based oscillators using nodal admittance matrix expansion. Analog Integrated Circuits and Signal Processing 1, 43-59 (2010)

10. AM Soliman et al., Applications of voltage and current unity gain cells in nodal admittance matrix expansion. Circuit and System Magazine 9(4), 29 (2009)

11. DG Haigh, PM Radmore, Admittance matrix models for the nullor using limit variables and their application to circuit design. IEEE Trans. Circuits Syst. I, Reg. Papers 53(10), 2214-2223 (2010)

12. DG Haigh, FQ Tan, C Papavassiliou, Systematic synthesis of active-RC circuit building-blocks. Anal. Integr. Circuits Signal Process, Netherlands 43(3), 297-315 (2005)

13. RA Saad, AM Soliman, A new approach for using the pathological mirror elements in the ideal representation of active devices. International Journal of Circuit Theory and Applications 38(3), 148-178 (2010)

\section{Submit your manuscript to a SpringerOpen ${ }^{\circ}$ journal and benefit from:}

- Convenient online submission

- Rigorous peer review

- Open access: articles freely available online

- High visibility within the field

- Retaining the copyright to your article

Submit your next manuscript at $>$ springeropen.com 